

\title{
Cooperative control of a gripped load by a team of quadrotors
}

\author{
H. Sayyaadi* and A. Soltani \\ School of Mechanical Engineering, Sharif University of Technology, Azadi Avenue, Tehran, P.O. Box 11155-9567, Iran.
}

Received 30 January 2017; received in revised form 17 February 2018; accepted 2 July 2018

\section{KEYWORDS}

Output tracking

control;

Cooperative

transport;

Gripped load;

Quadrotors;

Disturbance rejection.

\begin{abstract}
In this paper, an output tracking controller is proposed for cooperative transport of a gripped load by a team of quadrotors. The proposed control law requires the measurement of only four state variables: position and yaw angle of the system. Moreover, the controller provides rejection of step and ramp external force disturbances. In addition, the control basis vectors derived via optimization facilitate the real-time determination of quadrotors' control inputs. Numerical simulations show the effectiveness of the proposed control scheme and its superiority over formerly designed controllers for such systems.
\end{abstract}

(C) 2019 Sharif University of Technology. All rights reserved.

\section{Introduction}

Unmanned Aerial Vehicles (UAV) have widely attracted engineers and scientists in the past decade. These platforms can be used in military operations, search and rescue operations, urbanization, etc. To extend capabilities of UAVs, they should be capable of transporting payloads individually or cooperatively. Therefore, scientists have investigated the load transport problem by UAVs in many research articles. Studies in this field can be divided into two general categories: studies about cable-suspended loads [1-19] and researches about gripped loads [11,20-25]. Furthermore, in some of these studies, load is transported by an individual quadrotor [1-8,10,12-17,19,23,24], while, in some other researches $[9,11,18,20-22,25]$, cooperative transport of payloads by UAV teams has been investigated. Different control techniques have been implemented to control UAVs and payloads in these

*. Corresponding author. Tel.: +982166165682 E-mail addresses: Sayyaadi@sharif.edu (H. Sayyaadi); asoltani@mech.sharif.edu (A. Soltani) studies such as adaptive control [2,3,14], reinforcement learning $[4,5,12]$, geometric control [7-9,15,18], model predictive control [22], LQR [19,22], gain scheduling [23], PID control [23,24], and sliding mode control [25].

In this study, cooperative transport of a gripped load by a team of quadrotors is addressed. Output tracking control scheme is implemented to design a trajectory tracking controller such that only the measurement of position and yaw angle of the system is required. Furthermore, step and ramp external force disturbances are rejected out by the designed controller. Moreover, to calculate the control inputs of the crowded network of the quadrotors in real-time, proper control basis vectors are derived by optimization of a cost function.

This study is organized as follows. Section 2 describes the modeling of the system in detail. The controller scheme is proposed in Section 3. In Section 4, numerical simulations are performed to examine the designed control laws in a cooperative transport of a gripped load. Conclusions are stated in Section 5 .

\section{Dynamic model}

In this section, configuration, acting forces and mo- 
ments, and equations of motion of the system are presented. Moreover, practical assumptions about modeling of the system are introduced.

\subsection{System configuration}

In this study, a team of identical quadrotors is implemented to grip and transport the load. To represent the rotational motion of the system with respect to the inertial reference frame, a body fixed frame $B_{L}$ is attached to the center of mass of the load. Moreover, a body fixed frame is attached to each quadrotor $Q_{i}$. Quadrotors grip the load such that $Z_{Q_{i}}$ for each quadrotor is parallel to $Z_{B_{L}}$ (Figure 1 ).

Furthermore, moment of inertia matrix of each quadrotor in its body-fixed frame $J_{Q}$ is diagonal and $J_{Q_{x x}}=J_{Q_{y y}}$. Moment of inertia tensor of the load in its body-fixed frame, $J_{L}$, is diagonal, too. Coordinates of the center of mass of each quadrotor in the bodyfixed frame of the load are denoted by $x_{i}^{B_{L}}, y_{i}^{B_{L}}$, and $z_{i}^{B_{L}}$. In addition, the number of quadrotors is denoted by $n$. Quadrotors should grip the load such that:

$$
\sum_{i=1}^{n} x_{i}^{B_{L}}=0, \quad \sum_{i=1}^{n} y_{i}^{B_{L}}=0, \quad \sum_{i=1}^{n} x_{i}^{B_{L}} y_{i}^{B_{L}}=0
$$

Consequently, coordinates of the center of mass of the entire system in $B_{L}$ frame denoted by $x_{c}^{B_{L}}, y_{c}^{B_{L}}$, and $z_{c}^{B_{L}}$ satisfy $x_{c}^{B_{L}}=y_{c}^{B_{L}}=0$. Furthermore, axes of the body-fixed frame of the entire system, $B$, are chosen
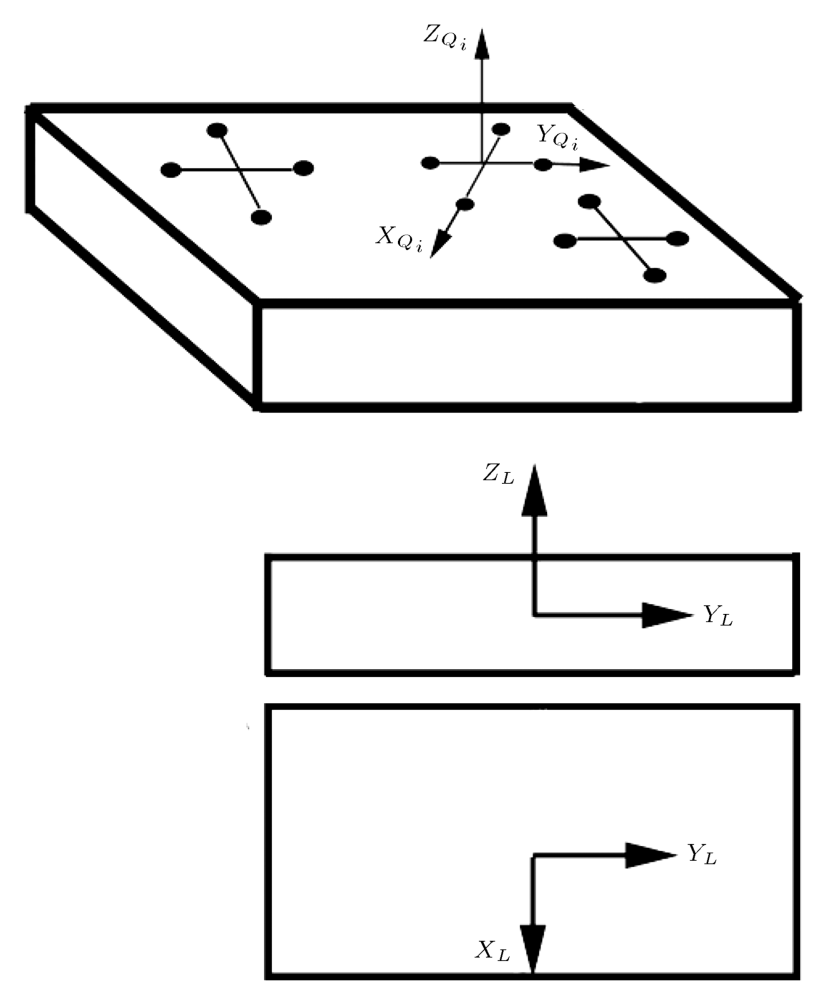

Figure 1. Body-fixed frames. parallel to those of $B_{L}$ so that:

$$
\sum_{i=1}^{n} x_{i}^{B}=0, \quad \sum_{i=1}^{n} y_{i}^{B}=0, \quad \sum_{i=1}^{n} x_{i}^{B} y_{i}^{B}=0,
$$

in which the coordinates of the center of mass of each quadrotor in $B$ frame are denoted by $x_{i}^{B}, y_{i}^{B}$, and $z_{i}^{B}$. Hence, the moment of inertia matrix of the entire system, $J$, will be diagonal, too. This matrix can be evaluated by the following equation accordingly:

$$
\begin{aligned}
J_{x x}= & J_{L_{x x}}+n J_{Q_{x x}}+m_{Q} \sum_{i=1}^{n}\left(y_{i}^{B}\right)^{2}+\left(z_{i}^{B}-z_{c}^{B}\right)^{2} \\
& +m_{L}\left(z_{c}^{B}\right)^{2} \\
J_{y y}= & J_{L_{y y}}+n J_{Q_{y y}}+m_{Q} \sum_{i=1}^{n}\left(x_{i}^{B}\right)^{2}+\left(z_{i}^{B}-z_{c}^{B}\right)^{2} \\
& +m_{L}\left(z_{c}^{B}\right)^{2}, \\
& \\
J_{z z}= & J_{L_{z z}}+n J_{Q_{z z}}+m_{Q} \sum_{i=1}^{N}\left(y_{i}^{B}\right)^{2}+\left(x_{i}^{B}\right)^{2} \\
J= & \operatorname{diag}\left(J_{x x}, J_{y y}, J_{z z}\right) .
\end{aligned}
$$

In Eq. (1), $m_{Q}$ and $m_{L}$ denote mass of each quadrotor and mass of the load, respectively. Moreover, $z_{L}^{B}$ represents $Z$ coordinate of the center of mass of the load in $B$ frame.

Obviously, total mass of the system can be evaluated as follows:

$$
m=n m_{Q}+m_{L}
$$

\subsection{Forces and moments}

Thrust force, $F$, and drag moment, $M_{z}$, produced by a rotor with an angular speed, $\omega$, can be approximated by:

$$
\begin{aligned}
& F=K_{F} \omega^{2}, \\
& M_{z}=K_{M} \omega^{2} .
\end{aligned}
$$

In Eq. (3), $K_{F}$ and $K_{M}$ are thrust and drag coefficients of the rotor. Moreover, drag moment acts in opposite direction of the rotor rotation direction. Each quadrotor has four rotors such that rotors number 1 to number 4 are on the positive $X_{Q_{i}}$, positive $Y_{Q_{i}}$, negative $X_{Q_{i}}$, and negative $Y_{Q_{i}}$, respectively. Furthermore, rotors number 1 and 3 rotate clockwise, while rotors number 2 and 4 rotate anti-clockwise. Distance from the axis of rotation of each rotor to the center of mass of the quadrotor is denoted by $L$. Therefore, net force and moments produced by rotors in $Q_{i}$ frame can be formulated as follows: 


$$
\begin{aligned}
{\left[\begin{array}{c}
F_{Q_{i}} \\
M_{x_{Q_{i}}} \\
M_{y_{Q_{i}}} \\
M_{z_{Q_{i}}}
\end{array}\right]=} & {\left[\begin{array}{cccc}
K_{F} & K_{F} & K_{F} & K_{F} \\
0 & K_{F} L & 0 & -K_{F} L \\
-K_{F} L & 0 & K_{F} L & 0 \\
-K_{M} & K_{M} & -K_{M} & K_{M}
\end{array}\right] } \\
& {\left[\begin{array}{c}
\omega_{i, 1}^{2} \\
\omega_{i, 2}^{2} \\
\omega_{i, 3}^{2} \\
\omega_{i, 4}^{2}
\end{array}\right] . }
\end{aligned}
$$

Consequently, according to the system configuration, total force and moments acting on the system can be calculated as follows:

$$
\begin{aligned}
{\left[\begin{array}{c}
F_{B} \\
M_{x_{B}} \\
M_{y_{B}} \\
M_{z_{B}}
\end{array}\right]=} & \sum_{i=1}^{n}\left[\begin{array}{cccc}
1 & 0 & 0 & 0 \\
y_{i}^{B} & \cos \left(\psi_{i}\right) & -\sin \left(\psi_{i}\right) & 0 \\
-x_{i}^{B} & \sin \left(\psi_{i}\right) & \cos \left(\psi_{i}\right) & 0 \\
0 & 0 & 0 & 1
\end{array}\right] \\
& {\left[\begin{array}{c}
F_{Q_{i}} \\
M_{x_{Q_{i}}} \\
M_{y_{Q_{i}}} \\
M_{z_{Q_{i}}}
\end{array}\right] }
\end{aligned}
$$

$\psi_{i}$ is the yaw angle of the quadrotor $Q_{i}$ with respect to $B$.

\subsection{Equations of motion}

Translational equations of motion of the system in the inertial reference frame can be formulated as follows:

$$
\ddot{r}=\frac{F_{B}}{m} R e_{3}-g e_{3},
$$

in which $r, g, e_{3}$, and $R$ are the position vector of the center of mass of the system, gravity acceleration, standard unit vector in $z$ direction, and rotation matrix from the inertial frame to $B$ frame.

Furthermore, equations of rotational motion of the system in $B$ frame are represented as the following equation by denoting angular velocity of the system by $\Omega$.

$$
J \dot{\Omega}=-\Omega \times J \Omega+\left[\begin{array}{c}
M_{x_{B}} \\
M_{y_{B}} \\
M_{z_{B}}
\end{array}\right] .
$$

\section{Controller design}

In this section, following the linearization of equations of motion with respect to the equilibrium point of the system, it is shown that translational equations of motion can be represented as a fourth-order linear system, while yaw dynamics of the system can be considered as a second-order linear system. Therefore, a fourthorder trajectory tracking controller and a second-order yaw controller are designed appropriately. Moreover, controller basis vectors are derived to determine control input of each quadrotor. Since the controller needs the measurement of position and yaw angle and, indeed, measurements made by motion capture systems are usually noisy, proper Kalman filters are introduced.

\subsection{Linearized dynamic model}

Rotation matrix $R$ can be represented by successive Yaw-Pitch-Roll rotations as in Eq. (8), shown in Box I.

In Eq. (8), $\phi, \theta$, and $\psi$ represent roll, pitch, and yaw angles, respectively. Therefore, equilibrium states of the system are obtained as follows:

$$
\begin{aligned}
& r=\dot{r}=0, \quad F_{B}=m g, \\
& \phi=\dot{\phi}=\theta=\dot{\theta}=\dot{\psi}=0 \\
& \psi=\psi_{e}
\end{aligned}
$$

According to the last line of Eq. (9), yaw angle of the system can be any desired value. Now, the linearized equation of motion with respect to the equilibrium point will be in the following form by assuming small angles approximation:

$$
\begin{aligned}
& \ddot{x}=g \cos \left(\psi_{e}\right) \theta+g \sin \left(\psi_{e}\right) \phi, \\
& \ddot{y}=g \sin \left(\psi_{e}\right) \theta-g \cos \left(\psi_{e}\right) \phi, \\
& \ddot{z}=\frac{F_{B}}{m}-g, \\
& \ddot{\phi}=\frac{M_{x_{B}}}{J_{x x}}, \quad \ddot{\theta}=\frac{M_{y_{B}}}{J_{y y}}, \quad \ddot{\psi}=\frac{M_{z_{B}}}{J_{z z}} .
\end{aligned}
$$

By taking the second derivative of the translational equations of motion with respect to time and combining them with the equations of rotational motion, one can find the following equations:

$$
R=\left[\begin{array}{ccc}
\cos (\theta) \cos (\psi) & \cos (\psi) \sin (\phi) \sin (\theta)-\cos (\phi) \sin (\psi) & \cos (\phi) \sin (\theta) \cos (\psi)+\sin (\phi) \sin (\psi) \\
\cos (\theta) \sin (\psi) & \sin (\psi) \sin (\phi) \sin (\theta)+\cos (\phi) \cos (\psi) & \cos (\phi) \sin (\theta) \sin (\psi)-\sin (\phi) \cos (\psi) \\
-\sin (\theta) & \cos (\theta) \sin (\phi) & \cos (\phi) \cos (\theta)
\end{array}\right]
$$




$$
\begin{aligned}
& x^{(4)}=g \cos \left(\psi_{e}\right) \frac{M_{y_{B}}}{J_{y y}}+g \sin \left(\psi_{e}\right) \frac{M_{x_{B}}}{J_{x x}}, \\
& y^{(4)}=g \sin \left(\psi_{e}\right) \frac{M_{y_{B}}}{J_{y y}}-g \cos \left(\psi_{e}\right) \frac{M_{x_{B}}}{J_{x x}}, \\
& z^{(4)}=\frac{\ddot{F}_{B}}{m}-g, \\
& \ddot{\psi}=\frac{M_{z_{B}}}{J_{z z}} .
\end{aligned}
$$

Consequently, equations of motion are simplified to:

$$
\begin{aligned}
& r^{(4)}=v, \\
& \ddot{\psi}=\frac{M_{z_{B}}}{J_{z z}},
\end{aligned}
$$

by following feedback linearization rule:

$$
\begin{aligned}
{\left[\begin{array}{c}
\ddot{F}_{B} \\
M_{x_{B}} \\
M_{y_{B}}
\end{array}\right]=} & {\left[\begin{array}{ccc}
J_{x x} \sin \left(\psi_{e}\right) / g & -J_{x x} \cos \left(\psi_{e}\right) / g & 0 \\
J_{y y} \cos \left(\psi_{e}\right) / g & J_{y y} \sin \left(\psi_{e}\right) / g & 0 \\
0 & 0 & m
\end{array}\right] } \\
& {\left[\begin{array}{l}
v_{x} \\
v_{y} \\
v_{z}
\end{array}\right]+\left[\begin{array}{l}
0 \\
0 \\
g
\end{array}\right] . }
\end{aligned}
$$

\subsection{Trajectory tracking controller design}

According to Eq. (13), to track the desired trajectory denoted by $r_{T}$, one can adopt $v$ as:

$$
\begin{aligned}
& v=-K_{p} e_{r}-K_{d} \dot{e}_{r}-K_{a} \ddot{e}_{r}-K_{j} e_{r}^{(3)}+r_{T}^{(4)} \\
& e_{r}=r-r_{T},
\end{aligned}
$$

in which $K_{p}, K_{d}, K_{a}$, and $K_{j}$ are positive constant gains. Therefore, dynamics of the trajectory tracking error for the linearized system will be as follows:

$$
e_{r}^{(4)}+K_{j} e_{r}^{(3)}+K_{a} \ddot{e}_{r}+K_{d} \dot{e}_{r}+K_{p} e_{r}=0 .
$$

To track the desired trajectory, it is sufficient for the gains of the controller to be chosen such that polynomial of Eq. (15) be Hurwitz. By this selection of the gains, trajectory tracking error will converge to zero exponentially.

Moreover, the equation of translational motion for the system subjected to external force disturbances can be represented as follows:

$$
\ddot{r}=\frac{F_{B}}{m} R e_{3}-g e_{3}+\frac{d(t)}{m},
$$

where $d(t)$ is the disturbance vector. Therefore, linearized equations of motion will be as follows:

$$
r^{(4)}=v+\frac{\ddot{d}(t)}{m} .
$$

By Eq. (14), trajectory tracking dynamics will be as follows:

$$
e_{r}^{(4)}+K_{j} e_{r}^{(3)}+K_{a} \ddot{e}_{r}+K_{d} \dot{e}_{r}+K_{p} e_{r}=\frac{\ddot{d}(t)}{m} .
$$

Therefore, steady-state tracking error is zero for step and ramp disturbances. In other words, step and ramp disturbances are rejected out by the controller properly.

\subsection{Yaw tracking controller}

To track the desired trajectory of yaw angle denoted by $\psi_{T}$ exponentially, it is sufficient to choose $M_{z_{B}}$ as follows:

$$
\begin{aligned}
& M_{z_{B}}=J_{z z}\left(-K_{p_{\psi}} e_{\psi}-K_{d_{\psi}} \dot{e}_{\psi}+\ddot{\psi}_{T}\right), \\
& e_{\psi}=\psi-\psi_{T} .
\end{aligned}
$$

In control law (Eq. (19)), $K_{p_{\psi}}$ and $K_{d_{\psi}}$ are positive constant gains.

\subsection{Control basis vectors}

Equation (5) defines four equations with $4 n$ unknowns and can be rewritten as follows:

$$
\left[\begin{array}{c}
F_{B} \\
M_{x_{B}} \\
M_{y_{B}} \\
M_{z_{B}}
\end{array}\right]=A u
$$

in which $A \in \mathbb{R}^{4 \times 4 n}$ is a constant matrix and can be determined from Eq. (5) by considering $u$ as:

$$
\begin{aligned}
u= & {\left[\begin{array}{lllll}
F_{Q_{1}} & M_{x_{Q_{1}}} & M_{y_{Q_{1}}} & M_{z_{Q_{1}}} \ldots F_{Q_{n}} & M_{x_{Q_{n}}} \\
& M_{y_{Q_{n}}} M_{z_{Q_{n}}}
\end{array}\right]^{T} . }
\end{aligned}
$$

For a system with more than one quadrotor, Eq. (20) remains undetermined. Therefore, one can choose $4 n-4$ of inputs independent of the desired net force and moments. However, an optimal control input $u^{*}$ that achieves the desired inputs while minimizing a cost function $J_{0}$ is adopted in this study. In other words,

$$
\begin{aligned}
& u^{*}=\arg \min _{u}\left\{J_{0} \mid\left[\begin{array}{llll}
F_{B}^{\text {des }} & M_{x_{B}}^{\text {des }} & M_{y_{B}}^{\text {des }} & M_{z_{B}}^{\text {des }}
\end{array}\right]^{T}\right. \\
& =A u\}
\end{aligned}
$$

where:

$$
\begin{aligned}
J_{0}= & \sum_{i=1}^{n} W_{F_{Q_{i}}} F_{Q_{i}}^{2}+W_{M_{x_{Q_{i}}}} M_{x_{Q_{i}}}^{2}+W_{M_{y_{Q_{i}}}} M_{y_{Q_{i}}}^{2} \\
& +W_{M_{z_{Q_{i}}}} M_{z_{Q_{i}}}^{2}
\end{aligned}
$$


In other words, $J_{0}=|H u|^{2}$ in which:

$$
\begin{gathered}
H=\operatorname{diag}\left(\sqrt{W_{F_{Q_{1}}}}, \sqrt{W_{M_{x} Q_{1}}}, \sqrt{W_{M_{y_{Q_{1}}}}}, \sqrt{W_{M_{z_{Q_{1}}}}},\right. \\
\left.\quad \ldots, \sqrt{W_{F_{Q_{n}}}}, \sqrt{W_{M_{Q_{n}}}}, \sqrt{W_{M_{y_{Q_{n}}}}}, \sqrt{W_{M_{z_{Q n}}}}\right)_{(24} .
\end{gathered}
$$

Hence, the optimal control input will be:

$$
\begin{aligned}
u^{*}= & H^{-2} A^{T}\left(A H^{-2} A^{T}\right)^{-1} \\
& {\left[\begin{array}{llll}
F_{B}^{\text {des }} & M_{x_{B}}^{\text {des }} & M_{y_{B}}^{\text {des }} & M_{z_{B}}^{\text {des }}
\end{array}\right]^{T} . }
\end{aligned}
$$

It is supposed that $W_{F_{Q_{i}}}=W_{F}$ and $W_{M_{z_{Q_{i}}}}=W_{M_{z}}$ because quadrotors are identical. Moreover, roll and pitch moment weights are adopted as $W_{M_{x_{Q_{i}}}}=W_{M_{y_{Q_{i}}}}=$ $W_{M_{x y}}$, because quadrotors are axially symmetric and roll and pitch moments are treated in the same way. After some algebraic manipulations, one can show that:

$$
\begin{aligned}
u^{*}= & {\left[\begin{array}{llll}
U_{F} & U_{M_{x}} & U_{M_{y}} & U_{M_{z}}
\end{array}\right] } \\
& {\left[\begin{array}{llll}
F_{B}^{\text {des }} & M_{x_{B}}^{\text {des }} & M_{y_{B}}^{\text {des }} & M_{z_{B}}^{\text {des }}
\end{array}\right]^{T}, }
\end{aligned}
$$

$U_{F}, U_{M_{z}}, U_{M_{x}}$, and $U_{M_{y}}$ are obtained by Eq. (27) as shown in Box II.

Therefore, control basis vectors can be simply calculated through Eq. (27).

\subsection{Kalman filter}

In the case of implementing a motion capture system, position and yaw angle of the load can be measured by the markers of such systems. However, measurements may be noisy, which should be filtered. Moreover, higher order derivatives of position and yaw angle for the designed feedback controllers should be estimated from the measurements. Thus, one can use Kalman filters to estimate these states, because system (13) is observable and measurements usually are disturbed by zero mean Gaussian white noises.

\subsubsection{State transition and control-input models for the translational Kalman filter}

To estimate first to third derivatives of the position of the load for feedback, state transition and control input models of the Kalman filter should be considered respectively as follows:

$$
A_{r}=\left[\begin{array}{cccc}
1 & T_{s} & \frac{T_{s}^{2}}{2} & \frac{T_{s}^{3}}{6} \\
0 & 1 & T_{s} & \frac{T_{s}^{2}}{2} \\
0 & 0 & 1 & T_{s} \\
0 & 0 & 0 & 1
\end{array}\right], B_{r}=\left[\begin{array}{c}
\frac{T_{s}^{4}}{24} \\
\frac{T_{s}^{3}}{6} \\
\frac{T_{s}^{2}}{2} \\
T_{s}
\end{array}\right] .
$$

Sampling time is denoted in Eq. (28) by $T_{s}$ accordingly.

\subsubsection{State transition and control-input models for yaw angle Kalman filter}

State transition and control input models of the Kalman filter to estimate yaw angle and velocity from noisy measurements should be:

$$
A_{\psi}=\left[\begin{array}{cc}
1 & T_{s} \\
0 & 1
\end{array}\right], B_{\psi}=\left[\begin{array}{c}
\frac{T_{s}^{2}}{2} \\
T_{s}
\end{array}\right] .
$$

\section{Simulations}

In this section, the performance of the designed control laws in position and yaw angle trajectory tracking of a rectangular cubic load (Figure 2) with a team of four identical quadrotors is examined. Moreover, orientation and location of the body-fixed frame $B_{L}$ are demonstrated in Figure 3. Moreover, physical and geometrical properties of the load and quadrotors are listed in Table 1.

The body-fixed frame of the entire system should be located at the center of the mass of the system parallel to $B_{L}$ as described formerly. To validate the designed controller fairly, the nonlinear model of the system represented by Eqs. (4) to (7) is used in simulations. This model has been verified experimentally in [21].

$$
\begin{aligned}
& U_{F}=\frac{1}{n}\left[\begin{array}{lllllllll}
1 & 0 & 0 & 0 & \ldots & 1 & 0 & 0 & 0
\end{array}\right]^{T}, \\
& U_{M_{z}}=\frac{1}{n}\left[\begin{array}{lllllllll}
0 & 0 & 0 & 1 & \ldots & 0 & 0 & 0 & 1
\end{array}\right]^{T},
\end{aligned}
$$

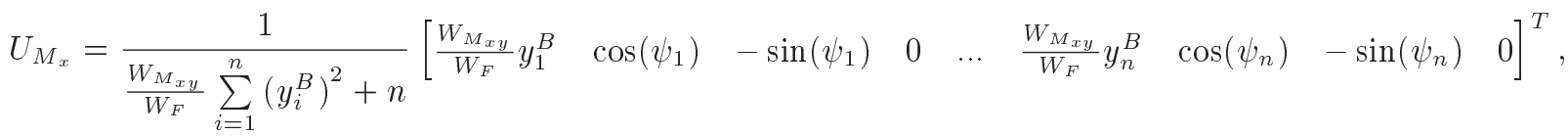

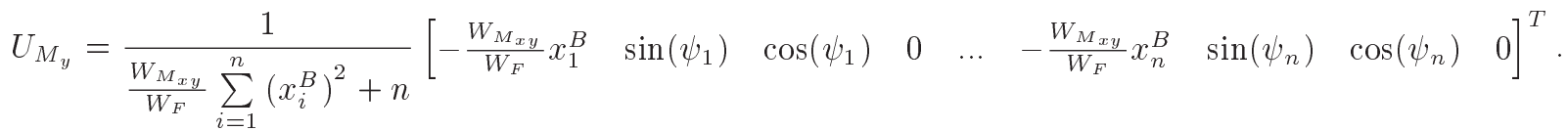




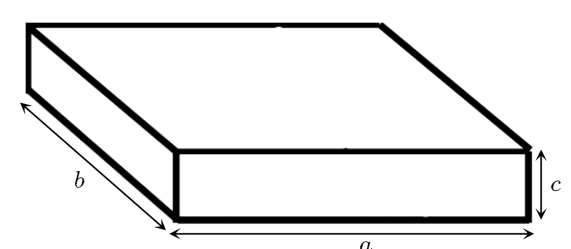

Figure 2. Load geometrical shape.

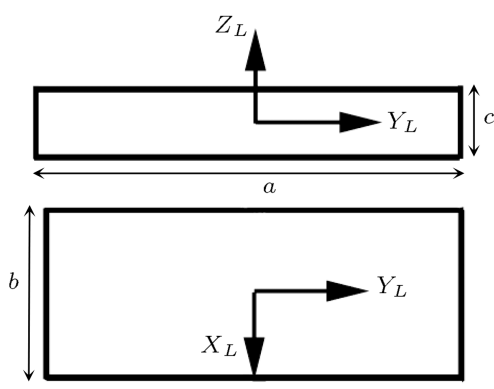

Figure 3. Direction and location of the body fixed frame of the load.

\subsection{Trajectory generation}

In the considered mission for the quadrotors, they should transport the load from its initial position to a desired destination at a given time. In addition, they should rotate the load to a final desired yaw angle. Therefore, proper trajectories should be generated for position and yaw angle of the system. According to Eq. (13), snap of the position trajectory and acceleration of the yaw trajectory should be minimized to have a minimum input trajectory. Consequently, trajectory generation for position of the system can be formulated as follows:

$$
\begin{aligned}
\min & \int_{t_{0}}^{t_{f}}\left|r_{T}^{(4)}(t)\right|^{2} d t \\
\text { s.t. } & \left\{\begin{array}{l}
r_{T}\left(t_{0}\right)=r_{0} \\
r_{T}^{(i)}\left(t_{0}\right)=0, i=1,2,3 \\
r_{T}\left(t_{f}\right)=r_{f} \\
r_{T}^{(i)}\left(t_{f}\right)=0, i=1,2,3
\end{array}\right.
\end{aligned}
$$

In this formulation, $t_{0}, t_{f}, r_{0}$, and $r_{f}$ are the initial time, given final time, initial position, and final desired position, respectively. By introducing a variable as $\tau=\frac{t-t_{0}}{t_{f}}$, trajectory generation can be rewritten as follows:

$$
\begin{aligned}
\min & \int_{0}^{1}\left|r_{T}^{(4)}(\tau)\right|^{2} d \tau \\
\text { s.t. } & \left\{\begin{array}{l}
r_{T}(0)=r_{0} \\
r_{T}^{(i)}(0)=0, i=1,2,3 \\
r_{T}(1)=r_{f} \\
r_{T}^{(i)}(1)=0, i=1,2,3
\end{array}\right.
\end{aligned}
$$

Now, trajectory generation can be formulated as a quadratic problem considering desired trajectory in each direction as a P-order polynomial of $\tau$. For example, trajectory generation in $X$ direction can be rewritten as the following quadratic problem:

$$
\begin{aligned}
& \min c_{x}^{T} H c_{x}, \\
& \text { s.t. } A_{x} c_{x}=B_{x},
\end{aligned}
$$

for the desired trajectory defined as follows:

$$
x_{T}=c_{0 x}+c_{1 x} \tau+c_{2 x} \tau^{2}+\ldots+c_{p x} \tau^{p} .
$$

In the quadratic form of the trajectory generation problem, vector $c_{x}$ is constructed as:

$$
c_{x}=\left[\begin{array}{llll}
c_{0 x} & c_{1 x} & \ldots & c_{p x}
\end{array}\right] .
$$

Moreover, matrix $H$ can be evaluated by calculating the following integral.

$$
\begin{aligned}
& H=\int_{0}^{1} h^{T}(\tau) h(\tau) \cdot d \tau \\
& h(\tau)=\left[\begin{array}{lllllll}
0 & 0 & 0 & 0 & 24 & 120 \tau & 360 \tau^{2} \\
& \quad \ldots & p(p-1)(p-2)(p-3) \tau^{p-4}
\end{array}\right] \otimes I_{3 \times 3} .
\end{aligned}
$$

Table 1. Physical and geometrical properties of the system.

\begin{tabular}{cccc}
\hline$m_{L}$ & $0.2 \mathrm{Kg}$ & $x_{1}^{B_{L}}$ & $-0.3 \mathrm{~m}$ \\
$m_{Q}$ & $0.65 \mathrm{Kg}$ & $x_{2}^{B_{L}}$ & $0.3 \mathrm{~m}$ \\
$J_{Q}$ & $\operatorname{diag}(7.5,7.5,13) \times 10^{-3} \mathrm{~kg} \cdot \mathrm{m}^{2}$ & $x_{3}^{B_{L}}=x_{4}^{B_{L}}$ & 0 \\
$a$ & $1.5 \mathrm{~m}$ & $y_{1}^{B_{L}}=y_{2}^{B_{L}}$ & $0.45 \mathrm{~m}$ \\
$b$ & $0.8 \mathrm{~m}$ & $y_{3}^{B_{L}}$ & $-0.7 \mathrm{~m}$ \\
$c$ & $0.01 \mathrm{~m}$ & $y_{4}^{B L}$ & $-0.2 \mathrm{~m}$ \\
$J_{x x}$ & $0.1845 \mathrm{~kg} \cdot \mathrm{m}^{2}$ & $\psi_{1}=\psi_{2}$ & 0 \\
$J_{y y}$ & $0.6753 \mathrm{~kg} \cdot \mathrm{m}^{2}$ & $\psi_{3}=\psi_{4}$ & $\frac{\pi}{4}$ \\
$J_{z z}$ & $0.1071 \mathrm{~kg} \cdot \mathrm{m}^{2}$ & $z_{i}^{B_{L}}, i=1,2,3,4$ & $0.105 \mathrm{~m}$ \\
\hline
\end{tabular}


Furthermore, matrices $A_{x}$ and $B_{x}$ are constructed as:

$$
\begin{aligned}
& A_{x}=\left[\begin{array}{l}
A_{0 x} \\
A_{f x}
\end{array}\right], \\
& B_{x}=\left[\begin{array}{l}
B_{0 x} \\
B_{f x}
\end{array}\right],
\end{aligned}
$$

in which:

$$
\begin{aligned}
A_{0 x} & =\left[\begin{array}{cccccc}
1 & 0 & 0 & 0 & \ldots & 0 \\
0 & 1 & 0 & 0 & \ldots & 0 \\
0 & 0 & 2 & 0 & \ldots & 0 \\
0 & 0 & 0 & 6 & \ldots & 0
\end{array}\right], \quad B_{0 x}=\left[\begin{array}{c}
x_{0} \\
0 \\
0 \\
0
\end{array}\right], \\
A_{f x} & =\left[\begin{array}{cccccc}
1 & 1 & 1 & 1 & \ldots & 1 \\
0 & 1 & 2 & 3 & \ldots & p \\
0 & 0 & 2 & 6 & \ldots & p(p-1) \\
0 & 0 & 0 & 6 & \ldots & p(p-1)(p-2)
\end{array}\right], \\
B_{f x} & =\left[\begin{array}{c}
x_{f} \\
0 \\
0 \\
0
\end{array}\right] .
\end{aligned}
$$

This procedure can be applied in other directions similarly. As mentioned before, the acceleration of the yaw trajectory should be minimized. Therefore, trajectory generation for yaw angle is an optimization problem as follows:

$$
\begin{gathered}
\min \int_{t_{0}}^{t_{f}}\left|\psi_{T}^{(2)}(t)\right|^{2} d t \\
\text { s.t. }\left\{\begin{array}{l}
\psi_{T}\left(t_{0}\right)=\psi_{0} \\
\psi_{T}^{(1)}\left(t_{0}\right)=0 \\
\psi_{T}\left(t_{f}\right)=\psi_{f} \\
\psi_{T}^{(1)}\left(t_{f}\right)=0
\end{array}\right.
\end{gathered}
$$

This optimization problem can be formulated as a quadratic problem by the same variable change and considering yaw trajectory as a polynomial function of $\tau$. Problem formulation for yaw angle trajectory generation is similar to the position trajectory planning procedure; however, matrices are different according to the optimization constraints.

\subsection{Mission characteristics}

In the considered mission, trajectories should be generated such that quadrotors transport the system from the origin of the reference frame to the coordinate $\left[\begin{array}{lll}1 & 2 & 3\end{array}\right]^{T}(\mathrm{~m})$. Furthermore, the system is in equilibrium at the origin of the inertial frame initially. Moreover, the initial yaw angle is about $0.2 \mathrm{rad}$, which should be set to zero at the end of mission. Mission duration is considered to be about 20 seconds. Furthermore, sampling time is $0.01 \mathrm{~s}$ in the simulation.

\subsection{Tracking performance of the controller}

Controller gains are adopted by LQR design appropriately. Furthermore, position and yaw measurements are made noisy by white Gaussian zero-mean noises to examine the performance of the Kalman filters. Moreover, weighting parameters are adopted such that $\frac{W_{M_{x y}}}{W_{F}}=\frac{1}{2}$. This ratio determines the importance of minimizing the thrust force or roll and yaw moments of each quadrotor. The performance of the proposed control scheme in tracking the desired trajectory of the load is demonstrated in Figure 4. Furthermore, yaw tracking performance of the controller is depicted in Figure 5. As demonstrated in these figures, although the controller designed by the linearized dynamic model of the system and the measurements is noisy, the desired trajectory has been tracked well by the proposed controller. Therefore, the designed controller along with Kalman filters can guarantee the stability of the system on the desired trajectories. Obviously, the estimation of the states by both of the filters is satisfactory because trajectory tracking aim is achieved. However, noisy measurements and filtered measurements are plotted in Figures 6 and 7 to
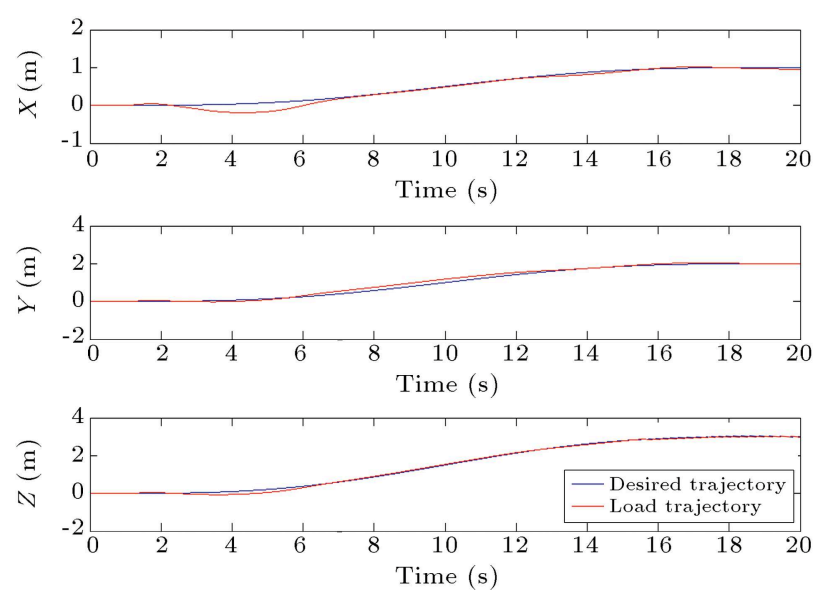

Figure 4. Trajectory of the load versus the desired trajectory.

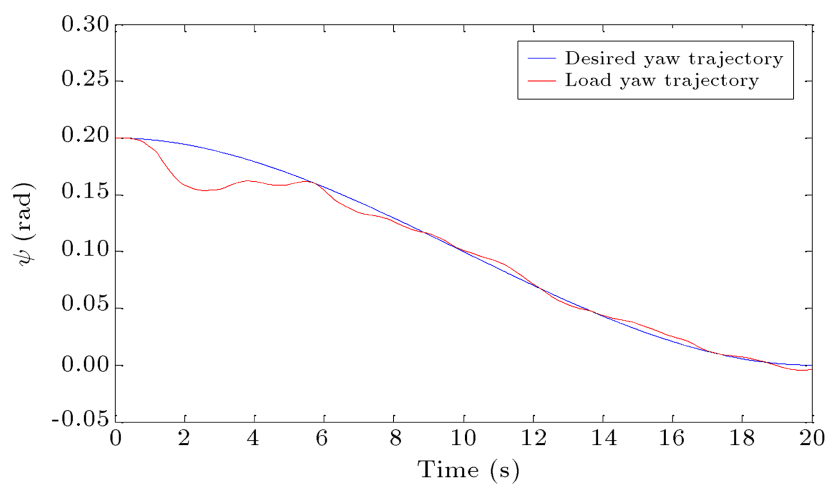

Figure 5. Yaw trajectory of the load versus the desired yaw trajectory. 

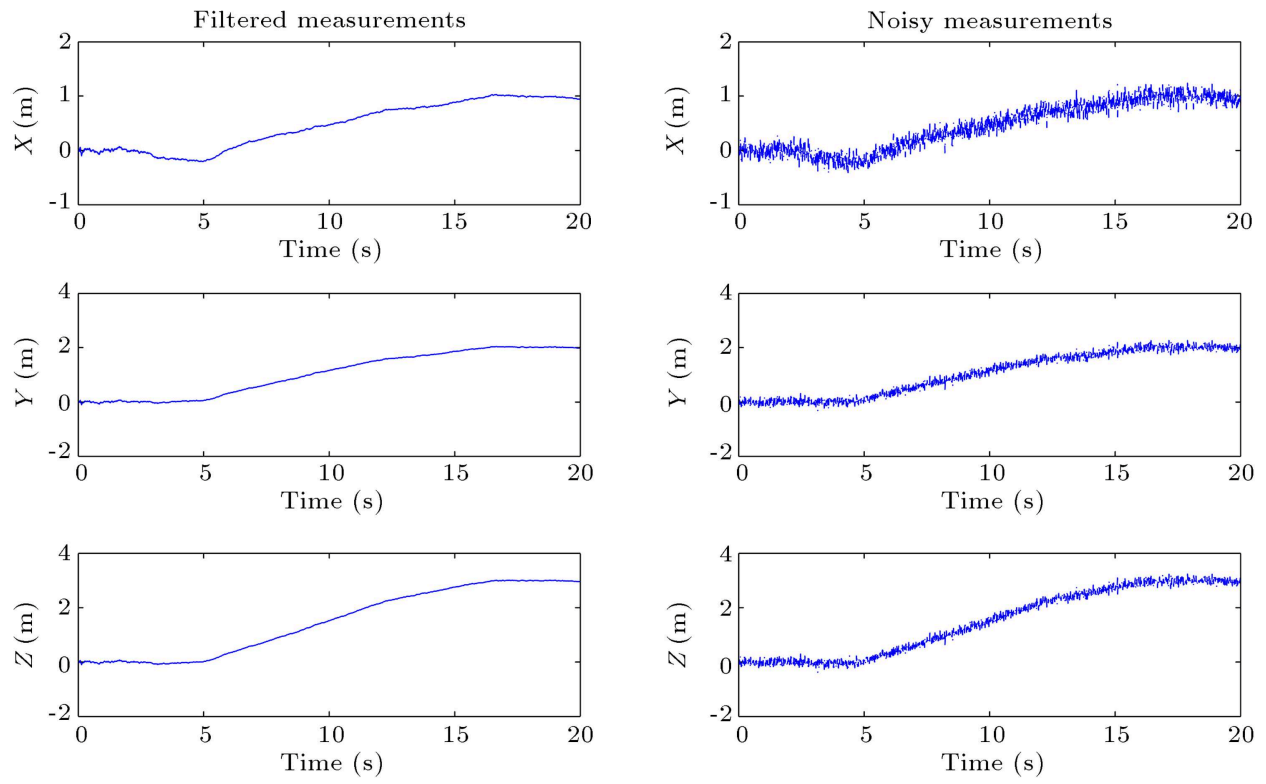

Figure 6. Noisy and filtered position measurements.
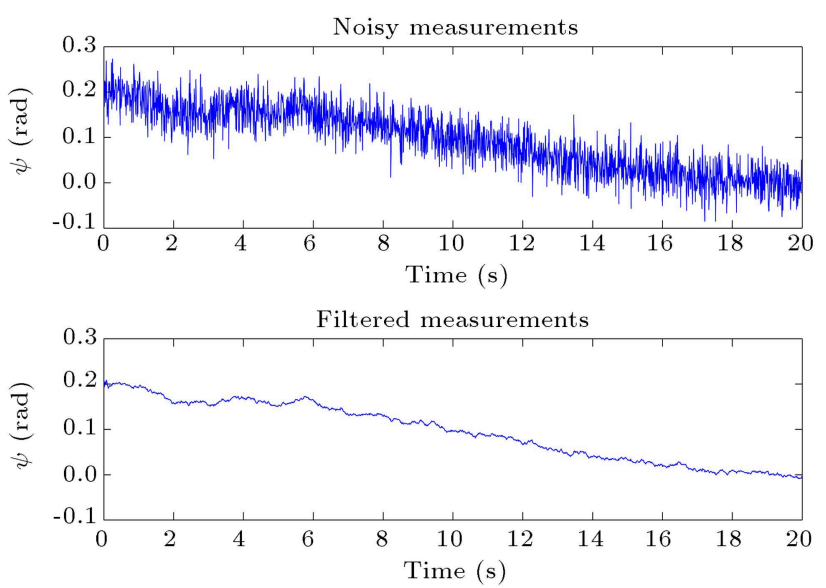

Figure 7. Noisy and filtered yaw measurement.

exhibit the performance of the position Kalman filter accordingly. Therefore, the measurement of position and yaw angle is sufficient for load trajectory tracking control by means of Kalman filtering.

\subsection{Disturbance rejection performance of the controller}

In the next simulations, the system is subjected to step and ramp force disturbances to examine disturbance rejection capability of the controller. At first, disturbance of the system is considered as $d(t)=$ $\left[\begin{array}{lll}5.6 & -2.8 & 8.4\end{array}\right]^{T}$. As depicted in Figure 8, tracking error converges to zero properly, and disturbance effect is attenuated by the proposed controller well. However, the controller of the study [21] has not rejected the disturbance, and the steady-state tracking error is about $1 \mathrm{~m}$.

Moreover, the system is also subjected to a ramp

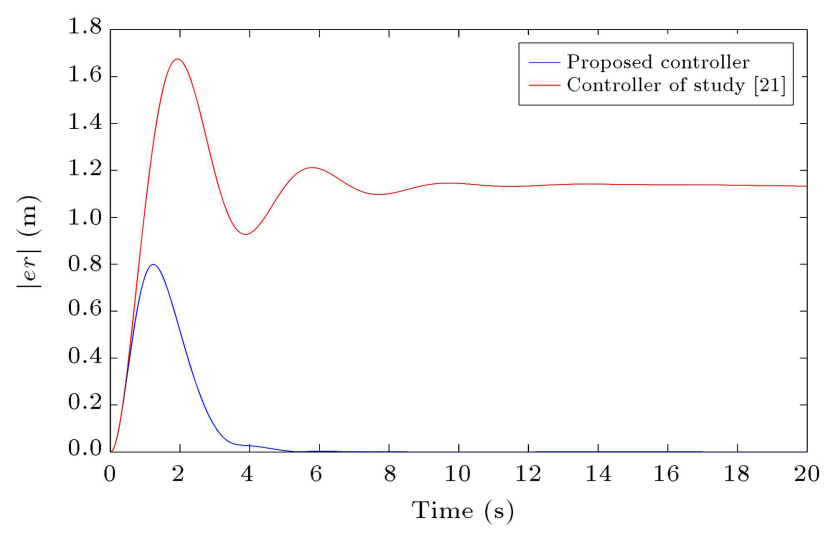

Figure 8. Trajectory tracking error in the presence of step disturbance by the proposed controller in this study and controller of study [21].

disturbance $d(t)=\frac{\left[\begin{array}{lll}5.6 t & -2.8 t & 8.4 t\end{array}\right]^{T}}{100}$ and performances of the controllers are compared accordingly. Figure 9 demonstrates that the proposed controller in this study has attenuated the disturbance properly while the controller of study [21] is unable to reject the disturbance.

Therefore, the proposed controller is more proper than formerly designed control laws to implement in real applications in which the considered system may be subjected to force disturbances such as wind.

\section{Conclusion}

In this paper, the performance of an output tracking controller is demonstrated on a challenging problem, i.e., cooperative transport of a gripped load by quadrotors. Simulations and analytical results illustrate that: 

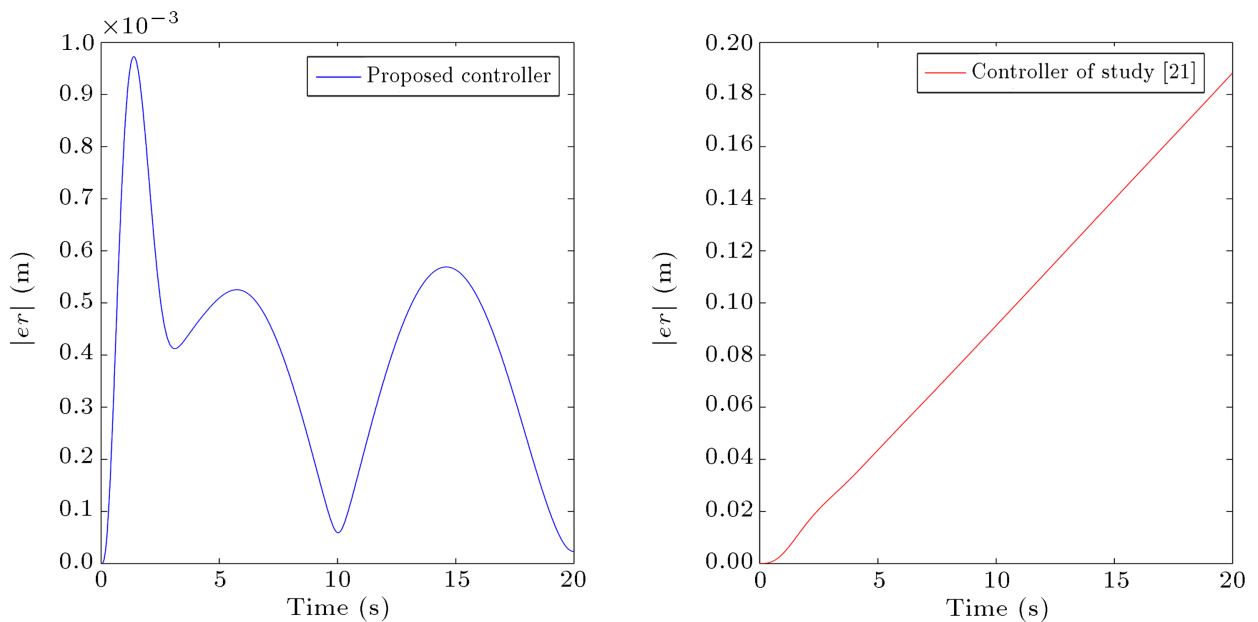

Figure 9. Trajectory tracking error in the presence of ramp disturbance by the proposed controller in this study and controller of study [21].

- The proposed controller provides a significant reduction in the disturbance influence on the tracking performance. Such capability of the controller is important in real applications where the effect of wind can cause problems;

- The designed control needs the measurement of only position and yaw angle of the system, which can be measured by motion capture systems such as Vicon in indoor applications;

- By applying the derived control basis vectors, realtime implementation of the control becomes possible.

The main drawback of the proposed control scheme is the required high bandwidth in the case of large networks. However, a similar centralized controller has been implemented to control gripped loads by four quadrotors in a study [21]. In future studies, the proposed controller will be implemented experimentally. In addition, a decentralized control scheme will be designed.

\section{References}

1. Bisgaard, M., la Cour-Harbo, A., Johnson, E.N., and Bendtsen, J.D. "Vision aided state estimator for helicopter slung load system", IFAC Proceedings Volumes, 40(7), pp. 425-430 (2007).

2. Cruz, P. and Fierro, R. "Autonomous lift of a cablesuspended load by an unmanned aerial robot", IEEE Conference on Control Applications (CCA), pp. 802807 (2014).

3. Dai, S., Lee, T., and Bernstein, D.S. "Adaptive control of a quadrotor UAV transporting a cable-suspended load with unknown mass", 53rd IEEE Conference on Decision and Control, pp. 6149-6154 (2014).
4. Faust, A., Palunko, I., Cruz, P., Fierro, R., and Tapia, L. "Learning swing-free trajectories for UAVs with a suspended load", IEEE International Conference on Robotics and Automation, pp. $4902-4909$ (2013).

5. Faust, A., Palunko, I., Cruz, P., Fierro, R., and Tapia, L. "Automated aerial suspended cargo delivery through reinforcement learning", Artificial Intelligence, 247, pp. 381-398 (2014).

6. Feng, Y., Rabbath, C.A., and Su, C.-Y. "Modeling of a micro UAV with slung payload", In Handbook of Unmanned Aerial Vehicles, K.P. Valavanis and G.J. Vachtsevanos, Eds., pp. 1257-1272, Springer Netherlands, Dordrecht (2015).

7. Goodarzi, F.A., Lee, D., and Lee, T. "Geometric stabilization of a quadrotor UAV with a payload connected by flexible cable", American Control Conference, pp. 4925-4930 (2014).

8. Goodarzi, F.A., Lee, D., and Lee, T. "Geometric control of a quadrotor UAV transporting a payload connected via flexible cable", International Journal of Control, Automation and Systems, 13(6), pp. 14861498 (2015).

9. Lee, T., Sreenath, K., and Kumar, V. "Geometric control of cooperating multiple quadrotor UAVs with a suspended payload", 52nd IEEE Conference on Decision and Control, pp. 5510-5515 (2013).

10. Notter, S., Heckmann, A., McFadyen, A., and Gonzalez, F. "Modelling, simulation and flight test of a model predictive controlled multirotor with heavy slung load", IFAC-Papers OnLine, 49(17), pp. 182-187 (2016).

11. Palunko, I., Cruz, P., and Fierro, R. "Agile load transportation : Safe and efficient load manipulation with aerial robots", IEEE Robotics \& Automation Magazine, 19(3), pp. 69-79 (2012).

12. Palunko, I., Faust, A., Cruz, P., Tapia, L., and Fierro, R. "A reinforcement learning approach towards 
autonomous suspended load manipulation using aerial robots", IEEE International Conference on Robotics and Automation, pp. 4896-4901 (2013).

13. Palunko, I., Fierro, R., and Cruz, P. "Trajectory generation for swing-free maneuvers of a quadrotor with suspended payload: A dynamic programming approach", IEEE International Conference on Robotics and Automation, pp. 2691-2697 (2012).

14. Potter, J.J., Adams, C.J., and Singhose, W. "A planar experimental remote-controlled helicopter with a suspended load", IEEE/ASME Transactions on Mechatronics, 20(5), pp. 2496-2503 (2015).

15. Sreenath, K., Lee, T., and Kumar, V. "Geometric control and differential flatness of a quadrotor UAV with a cable-suspended load", 52nd IEEE Conference on Decision and Control, pp. 2269-2274 (2013).

16. Sreenath, K., Michael, N., and Kumar, V. "Trajectory generation and control of a quadrotor with a cablesuspended load - A differentially-flat hybrid system", IEEE International Conference on Robotics and Automation, pp. 4888-4895 (2013).

17. Tang, S. and Kumar, V. "Mixed Integer Quadratic Program trajectory generation for a quadrotor with a cable-suspended payload", IEEE International Conference on Robotics and Automation (ICRA), pp. 22162222 (2015).

18. Wu, G. and Sreenath, K. "Geometric control of multiple quadrotors transporting a rigid-body load", 53rd IEEE Conference on Decision and Control, pp. 61416148 (2014).

19. Wu, G. and Sreenath, K. "Variation-based linearization of nonlinear systems evolving on $\mathrm{SO}(3)$ and $\mathrm{S}^{2}$, IEEE Access, 3, pp. 1592-1604 (2015).

20. Lindsey, Q., Mellinger, D., and Kumar, V. "Construction with quadrotor teams", Autonomous Robots, 33(3), pp. 323-336 (2012).

21. Mellinger, D., Shomin, M., Michael, N., and Kumar, V. "Cooperative grasping and transport using multiple quadrotors", In Distributed Autonomous Robotic Systems: The 10th International Symposium, A. Martinoli, F. Mondada, N. Correll, et al., Eds., pp. 545-558, Springer Berlin Heidelberg, Berlin, Heidelberg (2013).

22. Rubio, A.A., Seuret, A., Ariba, Y., and Mannisi, A. "Optimal control strategies for load carrying drones",
In Delays and Networked Control Systems, A. Seuret, L. Hetel, J. Daafouz, and K. H. Johansson, Eds., pp. 183-197, Springer International Publishing, Cham (2016).

23. Sadeghzadeh, I., Abdolhosseini, M., and Zhang, Y. "Payload drop application of unmanned quadrotor helicopter using gain-scheduled pid and model predictive control techniques", Intelligent Robotics and Applications, pp. 386-395 (2012).

24. Pounds, P.E.I., Bersak, D.R., and Dollar, A.M. "Stability of small-scale UAV helicopters and quadrotors with added payload mass under PID control", $A u$ tonomous Robots, 33(1), pp. 129-142 (2012).

25. Parra-Vega, V., Sanchez, A., Izaguirre, C., Garcia, O., and Ruiz-Sanchez, F. "Toward aerial grasping and manipulation with multiple UAVs", Journal of Intelligent \& Robotic Systems, 70(1), pp. 575-593 (2013).

\section{Biographies}

Hassan Sayyaadi received his BSc degree from Amirkabir University of Technology, Tehran, Iran, 1987, his MSc degree from Sharif University of Technology, Tehran, Iran, 1990, his PhD degree from the University of Tokyo, Japan, 2001, all in Mechanical Engineering. From 1990 to 1996, he was with the School of Mechanical Engineering of Sharif University of Technology and, from 2001, after his PhD studies, he has been again with the same school and university. Now, he is a Professor and his research interests include dynamics and control, robotics and mechanisms, artificial intelligence, and neural networks.

Ali Soltani received his BSc, MSc and $\mathrm{PhD}$ degrees all from the School of Mechanical Engineering of Sharif University of Technology, Tehran, Iran, 2007, 2010, and 2017, respectively, all in Mechanical Engineering. Since 2017, he has been with the Mechanical Engineering Department of Sahand University of Technology and his researches are all about multi-agent robotic systems and quadrotors. 